\title{
Virtual Reality for Vestibular Rehabilitation
}

\author{
Jae-Jun Song (10 \\ Department of Otorhinolaryngology-Head and Neck Surgery, Korea University College of Medicine, Seoul, Korea
}

With population aging and interest in improving the quality of life, focus on dizziness in the field of ear nose and throat has increased. A local study found that $21.7 \%$ of adults had experienced dizziness in the past year and that dizziness is a major risk factor for falls [1]. Diagnosis and treatment of dizziness would become more important with population aging in the future.

Vestibular rehabilitation is a treatment that, through exercise, promotes vestibular compensation and provides replacement of sensory input and habituation of the motion or situation that causes dizziness, which enhances postural and movement stability, reduces fall risk, and improves associated anxiety and depression. The recently developed customized vestibular rehabilitation therapy provides treatment by combining various exercises depending on the symptoms and conditions of the patient.

Virtual reality (VR) is an interface between humans and computers, which includes real-time simulation and interaction through various sensory channels, such as vision, hearing, touch, smell, and taste. The extent of the use of VR varies widely, and recent studies have appliedVR technologies in the medical field, including anatomy, three-dimensional patient modeling, virtual surgery, and rehabilitation. Rehabilitation systems using VR technologies have been studied and developed recently. In combination with wearable computers, VR can have a wide variety of applications. Further, by applying content such as games to continuously motivate patients to undergo rehabilitation and by making the treatment enjoyable and engaging, the effectiveness can be increased. Currently, studies combining rehabilitation with VR are underway.

Studies have been conducted on the use of VR to enhance the efficiency of vestibular rehabilitation. Previously, a balance rehabilitation unit, force platform, and Nintendo Wii Fit were used, but recently, immersive equipment using the head-mount display (HMD) has been developed. Rosiak et al. [2] showed that motion trackers and force-plate platforms, which are referred to as hybrid VR units, were effective for subjective reduction of symptoms in patients with peripheral vestibular dysfunction.
Micarelli et al. [3] showed that the home-based VR protocol using HMD can be a safe option to improve vestibular ocular reflex, postural control, and quality of life in patients with vestibular impairment in whom cognitive decline could hinder rehabilitation. Furthermore, Park et al. [4] showed that the VR device combined with eye trackers and HMD can improve goal-oriented attention and activation of brain networks while providing vestibular rehabilitation.

These VR devices employ games, unlike conventional equipment, which are enjoyable, increase concentration, and result in continuing treatment by the patient. Moreover, stimulating multiple senses simultaneously could increase the effectiveness of rehabilitation and help treat patients with visual vertigo. Early models of VR devices could not be commercialized because of expensive equipment and large locations, but recently, training using relatively inexpensive and simple equipment, such as HMD, seemed effective, which is likely to be more practical in the future and provide great help to full-scale rehabilitation.

\section{CONFLICT OF INTEREST}

No potential conflict of interest relevant to this article was reported.

\section{ORCID}

Jae-Jun Song https://orcid.org/0000-0002-8488-9091

\section{REFERENCES}

1. Chang J, Hwang SY, Park SK, Kim JH, Kim HJ, Chae SW, et al. Prevalence of dizziness and associated factors in South Korea: a crosssectional survey from 2010 to 2012. J Epidemiol. 2018 Apr;28(4): 176-84.

2. Rosiak O, Krajewski K,Woszczak M, Jozefowicz-Korczynska M. Eval-

Copyright (C) 2019 by Korean Society of Otorhinolaryngology-Head and Neck Surgery.

This is an open-access article distributed under the terms of the Creative Commons Attribution Non-Commercial License (http://creativecommons.org/licenses/by-nc/4.0)

which permits unrestricted non-commercial use, distribution, and reproduction in any medium, provided the original work is properly cited. 
uation of the effectiveness of a Virtual Reality-based exercise program for Unilateral Peripheral Vestibular Deficit. J Vestib Res. 2018; 28(5-6):409-15.

3. Micarelli A, Viziano A, Micarelli B, Augimeri I, Alessandrini M. Vestibular rehabilitation in older adults with and without mild cognitive impairment: effects of virtual reality using a head-mounted display. Arch Gerontol Geriatr. 2019 Jul-Aug;83:246-56.

4. Park JH, Jeon HJ, Lim EC, Koo JW, Lee HJ, Kim HJ, et al. Feasibility of eye tracking assisted vestibular rehabilitation strategy using immersive virtual reality. Clin Exp Otorhinolaryngol. 2019 Nov;12(4): 376-84.

Received June 25, 2019 Accepted August 23, 2019 